\title{
Erratum to: Human Guidance: Suggesting Walking Pace Under Manual and Cognitive Load
}

Tommaso Lisini Baldi, Gianluca Paolocci, and

Domenico Prattichizzo

\section{Erratum to: \\ Chapter "Human Guidance: Suggesting Walking Pace Under Workload" in: D. Prattichizzo et al. (Eds.): Haptics: Science, Technology, and Applications, LNCS 10894, https://doi.org/10.1007/978-3-319-93399-3_36}

The original version of this chapter contained an error in the title. The title has been changed from "Human Guidance: Suggesting Walking Pace Under Workload" to "Human Guidance: Suggesting Walking Pace Under Manual and Cognitive Load". 